\title{
TELEVISIÓN Y ADOLESCENCIA: VALORES Y ASPIRACIONES ACADÉMICO-PROFESIONALES
}

\author{
TELEVISION AND ADOLESCENCE: ACADEMIC AND \\ PROFESSIONAL ASPIRATIONS AND VALUES
}

\author{
Sonia Reigosa York* \\ CEIP “Joaquín Costa” de Tarazona
}

\begin{abstract}
RESUMEN
Con este estudio se pretende descubrir si en las elecciones o aspiraciones profesionales ha podido influir la forma en que muestran estas cuestiones los programas de televisión más vistos por los jóvenes en la actualidad. Hemos utilizado un diseño apriorístico de encuestación administrado a 59 adolescentes de un Instituto Público situado en la periferia de Zaragoza, España. Entre las conclusiones más destacadas encontramos que los adolescentes tienen varios propósitos como telespectadores y que son coherentes en sus opiniones en torno a sus aspiraciones y valores profesionales. Igualmente, descubrimos la existencia de un juicio crítico como televidentes, reflejado en su conciencia acerca de la posible influencia que ejerce el medio televisivo tanto en sí mismos como en los demás.
\end{abstract}

Palabras clave: Aspiraciones y valores profesionales, series televisivas, adolescentes.

\begin{abstract}
This study intends to find out if the TV programmes that are most watched by young people nowadays may have had an influence on their career choices or expectations. We have administered a survey to 59 adolescents of a Public Institute located in Zaragoza, Spain. Among the most outstanding conclusions we found that adolescents have several purposes, as viewers and that they are coherent with these opinions about their aspirations and values. We also discovered the existence of "a critical attitude" as viewers, reflected on their awareness of the possible influence that TV has both on themselves and on the others.
\end{abstract}

Key words: Career goals values, TV, adolescents.

\footnotetext{
* Sonia Reigosa York Licenciada en Psicopedagogía y diplomada en Magisterio de Audición y Lenguaje por la Universidad de Zaragoza. Ha obtenido el Diploma de Estudios Avanzados en el área de Métodos de Investigación y Diagnóstico en Educación" por la Universidad de Zaragoza. Es maestra interina de Audición y Lenguaje en el CEIP “Joaquín Costa” de Tarazona (Zaragoza). Forma parte de la Junta Directiva de la Asociación Aragonesa de Psicopedagogía, en concreto en la Vocalía de Formación. E-mail: sreigosayork@educaragon.org
} 


\section{Introducción}

"La televisión está ahí, delante de nosotros (...). Seguramente nada o casi nada en nuestra experiencia cotidiana ocupa tan insistentemente ese lugar, delante de nosotros, delante de nuestros ojos" (González, 1999:9). Es así como González Requena (1999) comienza su libro, y es así como nosotros entendemos este fenómeno que se ha impuesto en nuestras vidas como algo obvio, inocuo, cotidiano e interiorizado como es ver la televisión. Asimismo, estamos inmersos en un mundo cambiante y global donde las exigencias académicoprofesionales y los valores de los jóvenes no son los mismos que los de hace diez años o incluso menos. Hoy en día, el mercado laboral se caracteriza por:

1) Cambios en las tareas y naturaleza de los trabajos: de la fragmentación en el trabajo a una mayor polivalencia. Del predominio habilidades físicas y manuales al trabajo intelectual y altamente tecnológico.

2) Cambios en las estructuras organizativas: Pasan de ser jerárquicas y rígidas a una flexibilidad y autonomía por parte de sus miembros.

3) Revalorización del factor humano: relevancia de las personas y sus competencias.

4) Cambios en las necesidades de formación: nuevas cualificaciones y competencias que garanticen la competitividad en el mercado (Sánchez García, 2003: 223-234).

Este hecho, unido a otros muchos factores (como por ejemplo: incorporación de la mujer, retraso en el acceso al primer empleo, etc.), hace que las preferencias académico-profesionales y los valores profesionales de los jóvenes hayan cambiado, y es aquí donde queremos averiguar si en las elecciones de determinadas carreras universitarias o tipos de trabajos a los que se quiere acceder, ha podido influir la forma en que muestran estas cuestiones los programas de televisión más vistos por los jóvenes en la actualidad. Expertos en esta temática atribuyen a los medios de comunicación una importancia considerable en la socialización de valores, normas, actitudes y opiniones. Sánchez Noriega (1997: 149) señala dos condiciones que magnifican la capacidad de socialización del mensaje: a) cuando responde a expectativas, necesidades o intereses previos del grupo de referencia, y b) cuanto menos sea la experiencia directa del sujeto sobre un aspecto concreto de la realidad. Respecto al uso de los medios de comunicación por parte de los adolescentes, especialmente la televisión o Internet, algunos estudios señalan que cuando se les pide a los jóvenes que digan de qué fuentes obtienen información de interés para ellos, los porcentajes más altos los reciben la familia, los amigos y los medios de comunicación. Por detrás quedan los centros de enseñanza, los partidos políticos y las iglesias (Naval, C.; Lara, S.; Portilla, I.; Sadaba, Ch., 2002: 59). Además, destacan que, en general, los jóvenes desconfían de los medios de comunicación, se muestran críticos y los consideran manipuladores, especialmente a la televisión y a la prensa. Otros autores (INJUVE, 2000; Rodríguez et al, 2001; Naval et al, 2002; Sánchez, 1997; Vera Vila, 2005: 28) señalan que la televisión es el medio más utilizado, y que disminuye el peso de los programas informativos a la vez que aumenta la audiencia en teleseries. Nosotros nos centramos en los series televisivas actuales con mayor audiencia, no obstante, hay autores que han explorado otros tipos de programación, los llamados "reality shows" o programas de telerealidad como "Gran Hermano", "El Bus", "Supervivientes", "Operación Triunfo", etc. Aldea Muñoz (2004) realiza una investigación de encuestación a niños de educación infantil comprendidos entre los 3 y 6 años, motivado por la proliferación de los programas denominados "tele-basura". Entre sus conclusiones destacan la 
aceptación entre los niños de programas como "Operación Triunfo" y la compañía de adultos (padres) para ver la televisión.

Por otro lado, debemos pensar igualmente que los valores forman parte de un proceso más amplio y global como es el proceso de socialización. Vera Vila (2005: 19), en este sentido, reflexiona sobre la forma en la que intervienen en la construcción social de la identidad juvenil; sobre la imagen de la juventud que proyectan los medios y, por último, sobre el uso que los jóvenes hacen de ellos. Entre sus conclusiones merecen destacarse tres:

a) Los medios son capaces de complementar, potenciar o anular la influencia de otros agentes de socialización juvenil tan importantes como la familia o la escuela.

b) La educación de calidad es una de las herramientas más poderosas para mediar el impacto negativo que pueden tener los medios en el proceso de desarrollo de la juventud.

c) Las políticas culturales y educativas han de facilitar que los jóvenes de diferentes tendencias encuentren un espacio democrático para expresarse.

Medrano, Cortés y Palacios (2007) llevan a cabo un trabajo con el objetivo de crear una Escala de Valores Televisivos (Val-TV 0.1) basada en el modelo elaborado por Schwart. Estos autores parten de la hipótesis de que "los propios contenidos televisivos son fuente de aprendizaje de valores”. En Buckingham (1986: 613) estudió la audiencia joven de EastEnders, un serial que, en su primer año de emisión en la BBC se había convertido en el más popular de la televisión británica, especialmente entre los adolescentes. La conclusión general que extrae es que los jóvenes que participan en ella no son "víctimas" de la televisión ni absorben pasivamente su influencia (Montero, 2006: 102). En Dinamarca, Klitgaard (1996) llevó a cabo un estudio etnográfico con adolescentes de la serie Sensación de Vivir. Entre sus conclusiones observa un doble modo de relacionarse con la serie. Por un lado, los participantes realizaban una lectura caliente, mediante la proyección de sentimientos y la identificación con los personajes y situaciones, y, por el otro, una lectura fría, en la que se distanciaban y adoptaban una posición irónica. Pero estas dos posiciones, lejos de ser contradictorias, operaban de manera simultánea en buena parte de la audiencia (Klitgaard, 1996, Montero, 2006: 108). Montero (2006), por su parte, realiza un estudio empírico con adolescentes a partir del visionado de la conocida serie Al salir de clase. Esta autora reafirma que "la capacidad socializadora de los contenidos mediáticos se magnifica cuanto menor es la experiencia directa" (Montero, 2006, p. 177).

$\mathrm{Si}$ atendemos al género y cómo está representando en los modelos profesionales de las series televisivas, Freixas (2000) lleva a cabo un estudio en el que analiza estos aspectos, llegando a una serie de conclusiones que resumo a continuación:

1) Con relación con el tema de los estudios, notas y carreras profesionales, las mujeres aspiran más que los hombres a seguir estudiando y terminar los estudios. Igualmente, otorgan más importancia que ellos al hecho de conseguir unos buenos estudios, una carrera que les guste, acabar bien, etc. Los hombres otorgan una notable importancia al hecho de practicar un deporte de manera profesional y valoran más que ellas las carreras profesionales de militar, policía y bombero.

2) De manera general, las profesiones a las que aspiran hombres y mujeres son diferentes. Así, los chicos aspiran más que las chicas a ser empresarios, a estudiar carreras técnicas superiores y a aprender oficios y trabajos manuales. Ellas prefieren 
trabajos relacionados con el cuerpo, como por ejemplo ser modelo; se identifican más con oficios de turismo y relaciones públicas y con carreras como Medicina, Psicología, Fisioterapia, Matrona, etc.

3) Apenas hay diferencia respecto a las aspiraciones, tanto de hombres como mujeres, en el tema del trabajo/sueldo.

Asimismo, analizan anuncios televisivos y su relación con los modelos profesionales de género que reflejan, constatando que hombres y mujeres son presentados de manera contrapuesta. Así "los hombres:

a) tienen profesiones remuneradas;

b) llevan a cabo actitudes de poder y saber $y$

c) viven situaciones de riesgo y aventura.

Mientras que las mujeres:

a) llevan a cabo tareas domésticas;

b) están en la cocina;

c) realizan funciones nutrientes;

d) desempeñan un gasto altruista notable;

e) aparecen como seres estúpidos, simples y asustadizos;

f) se exhiben, se insinúan y desnudan" (Freixas, 2000: 7).

Por último vamos a analizar por qué aumenta la importancia de la escuela y de los medios audiovisuales en la educación en este medio. Pérez Tornero (2005: 3) considera que, entre otras cosas, la escuela y la televisión ocupan cada vez más tiempo en la vida de los jóvenes y de los niños. Entre posibles soluciones a esta situación propone:

1) Reforzar la función educativa de la familia.

2) Proteger la función educativa e instructiva de las escuelas.

3) Aumentar la responsabilidad educativa de la televisión.

4) Equilibrar los tiempos y las funciones entre familia-televisión-escuela.

Como reflexión sobre el estado de la cuestión aquí expuesto, podemos decir que, de acuerdo con Bermejo (2005; Medrano et al, 2007: 309), gran parte de los trabajos sobre televisión que hemos ido revisando se podrían clasificar dentro de un continuo. En uno de los polos se encontrarían las teorías que defienden la gran influencia de la televisión en la infancia y en el otro polo, por el contrario, se ubicarían aquellas que defienden que la televisión no ejerce una influencia.

\section{Método}

Hemos utilizado un diseño apriorístico de encuestación administrado a 59 adolescentes con edades comprendidas entre 14 y 19 años (27chicas y 32 chicos) de un Instituto Público situado en la periferia de Zaragoza, España. La selección de la muestra parte de la base de ser accesible. Esta investigación tiene un carácter descriptivo/exploratorio, no obstante, a partir de sus resultados y del estado de la investigación abordado, podremos establecer un conjunto de implicaciones de cara a mejorar la práctica educativa en torno a la educación en el medio 
televisivo de mayor difusión, como es la televisión. Para obtener la información requerida para nuestro estudio, hemos elaborado un cuestionario semiestructurado (en proceso de validación), en el que pretendemos obtener información en dos direcciones. Por un lado, en relación con sus preferencias televisivas: averiguar el tipo de personajes con los que se identifican (y los valores profesionales que creen que promueven), y por el otro, sus preferencias personales en relación a sus prioridades académico-laborales, y sus correspondientes valores profesionales. Señalar que dicho cuestionario está basado en la Escala de Valores Televisivos EVAT (Medrano, Palacios, Aierbe y Cortés, 2006) y en la tesis sobre las series juveniles y la formación de la identidad en la adolescencia realizada por Fraça (2001). Es en los meses de mayo-junio de 2007 cuando se procede a la recogida de datos mediante la administración del cuestionario a una muestra compuesta por estudiantes de $3^{\circ}-4^{\circ}$ de la E.S.O y $1^{\circ}$ Bachillerato. En los meses de verano (Julio-Septiembre) se procede a su análisis con la ayuda del paquete estadístico SPSS, y su posterior interpretación y obtención de conclusiones.

\section{Resultados}

A continuación vamos a ir exponiendo en función de las hipótesis y algunas preguntas de investigación de las que partíamos, los resultados más significativos:

1. El número de horas que dedican los adolescentes a ver la televisión no es excesivo.

El 54,2\% ve entorno a 3-4 horas diarias; el 35,6\% una o dos horas y el 10,2\% unas cinco o seis horas. Respecto a la edad, no encontramos diferencias significativas $(\mathrm{F}=.220$ $\mathrm{p}=.641)$ ni tampoco en relación al sexo $(\mathrm{F}=.776 \mathrm{p}=.382)$.

2. El visionado de la televisión cumple unos propósitos en la audiencia.

Entre las funciones que señalan como telespectadores giran entorno al Entretenimiento $(72,9 \%)$; seguida de diversión $(49,2 \%)$ y pasar el rato $(41,1 \%)$; informarme $(23,7 \%)$, para estar al día $(8,5 \%)$ y hablar con los amigos $(6,8 \%)$. El visionado de la televisión en todos nuestros encuestados cumple distintas funciones, incluso dentro de un mismo sujeto.

3. En el grupo de edad comprendido entre los 16-19 años existen menos dudas en cuanto a las elecciones académico-profesionales.

Encontramos una relación significativa entre el tramo de edad 16-19 años y el hecho de tener más claro a qué se quieren dedicar profesionalmente (Prueba de Chi Cuadrado, Sig. asintótica bilateral $=.005)$. Asimismo, hallamos que la mayoría de nuestros adolescentes saben a qué tipo de profesión quieren desempeñar en un futuro próximo (72,9\%). Respecto a las profesiones elegidas por nuestra muestra, encontramos una gran diversidad: soldador/a, psicólogo/a, abogado/a, arquitecto/a, filósofo/a, policía, maestra/o, enfermera/o, deportista, peluquera/o, carpintero, administrativo/a, veterinario/a, chapista, informático/a, dentista, ingeniero/a, etc. Por ello, hemos agrupado dichas profesiones en unas categorías para facilitar su análisis y posterior interpretación. Dichas categorías son las siguientes: profesiones sin cualificación, con cualificación, técnico grado medio y profesiones liberales con estudios superiores. La mayoría de nuestros alumnos $(32,2 \%)$ han elegido profesiones que exigen una cualificación (soldador, peluquero, policía, deportista, carpintero, ...), seguido de 
profesiones con estudios superiores (30,5\%), tales como: veterinario, maestro, abogado, arquitecto, psicólogo, etc. Y unos pocos $(10,2 \%)$ técnicos de grado medio (puericultor/a, cocinero, auxiliar de medicina, imagen personal, delineante, etc.).

4. Gustos y características personales juegan un papel importante a la hora de elegir una profesión.

Partíamos de la hipótesis de que los modelos profesionales serían tenidos en cuenta en la elección de las aspiraciones laborales de nuestra muestra, no obstante, la gran mayoría considera que en su elección profesional no ha tenido nada que ver los modelos profesionales que ofrecen las series televisivas $(84,7 \%)$. Entre las razones por las que han elegido dichas profesiones, encabeza la lista los gustos y características personales con un $45,8 \%$, seguido de padres y familiares cercanos $(16,9)$ el prestigio de la profesión y las salidas profesionales $(15,3 \%)$; posibilidades de éxito $(10,2 \%)$, profesorado $(5,1 \%)$ y en último lugar, los compañeros con un $1,7 \%$.

5. Los adolescentes eligen más aquellos personajes cuyos valores coincidan con los suyos propios.

Atendiendo a los valores laborales asociados a los personajes televisivos elegidos por nuestra muestra, en primer lugar nos encontramos con la satisfacción personal (20,3\%); seguido por el descubrimiento, indagar, investigar $(11,9 \%)$; sociabilidad $(8,6 \%)$; ganar dinero $(8,5 \%)$; independencia $(6,8 \%)$; competitividad $(6,8 \%)$; Creatividad $(5,1 \%)$, Altruismo $(5,1 \%)$; Seguridad (5,1\%); Dirección de otras personas $(1,7 \%)$; Ejecución de órdenes sin responsabilidades $(1,7 \%)$ y prestigio $(1,7 \%)$. Descubrimos que existe cierta coherencia entre los valores laborales de los protagonistas con los que se identifican y sus propios valores. Así, por ejemplo, vemos cómo en los primeros puestos se encuentran valores laborales tales como: sociabilidad (con un 49,2\% que está completamente de acuerdo y un $37,3 \%$ de acuerdo), le sigue satisfacción personal (44,1\% completamente de acuerdo y un $42,4 \%$ de acuerdo), ganar dinero (42,4\% está completamente de acuerdo y un $39 \%$ de acuerdo) e independencia (40,7\% está completamente de acuerdo y un 32,2\% de acuerdo). Entre las más penalizadas se encuentra Ejecución de órdenes sin responsabilidades (20,3\% completamente en desacuerdo y $20,3 \%$ en desacuerdo). Y con un porcentaje bastante amplio de indiferencia se sitúan dirección de otras personas (Indiferente un 49,2\% y en desacuerdo un $27,1 \%$ ), prestigio (40,7\% indiferente), y competitividad (37,3\% indiferente).

6. Más de la mitad de los encuestados ha aprendido algo viendo las series televisivas.

Más de la mitad de nuestros encuestados (52,5\%) considera que ha aprendido algo como telespectador de series televisivas. Sus comentarios han sido agrupados en varias categorías: consejos sobre la vida $(22 \%)$, valores personales $(8,5 \%)$, conocimientos sobre determinados temas $(6,8 \%)$, cómo actuar en determinadas situaciones $(3,4 \%)$ y comentarios/chistes $(3,4 \%)$.

\section{Conclusiones}

Dentro de los medios de comunicación, la televisión es el más accesible para los jóvenes, ya que está presente en la mayor parte de los hogares y no requiere de habilidades muy 
complejas para recibir la información. En este estudio nos preguntábamos acerca de la posible influencia que puedan ejercer los modelos profesionales de las series televisivas elegidas por nuestra muestra y su coincidencia con sus aspiraciones y valores laborales. Entre las conclusiones más destacadas encontramos que no existe una correspondencia entre los modelos laborales de los personajes con los que se identifica nuestra muestra y sus propias aspiraciones, incluso la mayoría ni siquiera se identifica con un personaje $(69,5 \%)$. Entre las posibles interpretaciones que podemos realizar en base a esto, en primer lugar, partimos de la base de que los telespectadores no juegan un papel pasivo en la recepción de los mensajes televisivos, sino todo lo contrario, existe una implicación activa de los espectadores en la producción de significado. En segundo lugar, cabe clarificar que, a diferencia de Buckingham (1996; Montero, 2006) que entre sus conclusiones llegó a la idea de que la identificación se producía con los personajes que eran como ellos, en nuestra muestra descubrimos que hay distintas interpretaciones respecto a lo que cada adolescente entiende por identificación con un personaje. Así pues, a partir de las explicaciones que nos ofrecen nuestros encuestados se crean tres categorías a partir de la clasificación que nos ofrecen distintos autores:

1) Identificación, el cual se refiere al sentimiento de unión con el personaje, a la impresión de experimentar su historia a través de sus ojos (Rubin y Perse, 1988; Montero, 2006: 133). Ej: “La relación de Sarita con Lucas se refleja en la mía con otro chico".

2) Identificación deseada, utilizada para denotar la intención de imitar al personaje, ya sea en general o en un aspecto concreto (Hoffner, 1996; ibidem, 133). Ej: "Porque quiero estudiar eso mismo y dedicarme profesionalmente".

3) Afinidad que consiste en la atracción sentida hacia un personaje, en definitiva, en si gusta o no (Newton y Buck, 1985; ibidem, 134). Ej: "Porque me gusta como actúa y su personalidad en la serie".

Con ello, reafirmamos la idea de que la recepción por parte de los adolescentes no es pasiva, y por tanto, no es unidireccional, sino bidireccional.

Siguiendo con los modelos profesionales, existe la creencia, incluso entre los propios adolescentes, de que la televisión ejerce una influencia importante en la creación de "modas" o en la demanda de determinadas profesiones. Por ejemplo, en Estados Unidos se ha comprobado que las profesiones de los protagonistas de las series de éxito se convirtieron en las más demandadas en muchas universidades, que vieron incrementar el número de matriculados coincidiendo con la popularidad de sus personajes (Ferrés, 1996; Montero, 2006: 24). Sin embargo, en nuestra muestra, la gran mayoría considera que en su elección profesional no ha tenido nada que ver los modelos profesionales que ofrecen las series televisivas $(84,7 \%)$. De hecho, entre las razones por las que han elegido dichas profesiones, encabeza la lista los gustos y características personales con un 45,8\%, seguido de padres y familiares cercanos $(16,9)$ el prestigio de la profesión y las salidas profesionales $(15,3 \%)$; posibilidades de éxito $(10,2 \%)$, profesorado $(5,1 \%)$ y en último lugar, los compañeros con un $1,7 \%$.

Atendiendo a los valores laborales, en el estudio elaborado recientemente por Medrano et al (2007) se reflexionaba en torno a la influencia de la televisión entre jóvenes y adolescentes a través de una encuesta elaborada en el País Vasco. Al igual que estos autores, consideramos que "los telespectadores ven más aquellos programas que coinciden con sus pro- 
pios valores" (Medrano et al, 2007, p. 137). Es por ello, que una de nuestras hipótesis es: los adolescentes elegirán aquellos personajes cuyos valores coincidan con los suyos propios. Los valores laborales asociados a los personajes televisivos van encaminados más a aspectos relacionados con la motivación intrínseca (satisfacción, descubrimiento, sociabilidad). Estos resultados coinciden con la afirmación de Montero (2006) acerca de que "en la mayoría de los casos, los encuestados se identificaron con un personaje con rasgos y comportamientos indicados como positivos" (Montero, 2006, p. 194). En nuestro caso, se identifican con valores considerados como positivos (satisfacción personal, descubrimiento, indagar, investigar, sociabilidad). Un aspecto no menos importante es el relativo a conocer si los propios adolescentes consideran que las series televisivas reflejan la realidad. Basándonos en el repaso a distintas investigaciones, y en concreto, en la realizada por Pasquier (1995) distingue tres tipos de relaciones con la serie Hélène et les garçons en función de la edad. Sin embargo, en nuestra muestra no encontramos diferencias en este aspecto en relación con la edad, e incluso, en general, la gran mayoría considera que los modelos laborales que ofrecen las series televisivas no coinciden con su desempeño real (71\%). Esto es un dato esclarecedor, ya que podemos afirmar que existe un juicio crítico como telespectador, e incluso esto mismo viene apoyado por las opiniones de nuestra muestra acerca de que la televisión les influye tanto a ellos mismos $(64,4 \%)$ como a sus compañeros $(66 \%)$. En ambos casos, consideran que les puede influir en mayor medida en el comportamiento (35\% y $32 \%$ respectivamente). Es decir, estos resultados ponen de manifiesto la existencia de un "filtro", de una actitud crítica a la hora de ver la variada parrilla televisiva que ofrecen los productores.

En cuanto a los propósitos de nuestra muestra como telespectadores, Aguaded (2000) señala que los adolescentes, en concreto, buscan una programación más específica que les sirva realmente, y no como una actividad solamente divertida, como lo suelen hacer los niños. Sin embargo, en nuestro estudio no se refleja dicha conclusión, ya que es cierto que el visionado de la televisión en todos nuestros encuestados cumple distintas funciones, incluso dentro de un mismo sujeto. No obstante, entre las funciones que señalan como telespectadores giran entorno al Entretenimiento (72,9\%), seguida de diversión (49,2\%) y pasar el rato $(41,1 \%)$. Esto resulta llamativo cuando más de la mitad de nuestros encuestados $(52,5 \%)$ considera que ha aprendido algo como telespectador de series televisivas, lo cual corroboraría en parte la conclusión de Aguaded acerca de que los adolescentes buscan una programación que les sirva realmente. No obstante, también se puede interpretar considerando que ambas cuestiones no son excluyentes, y que al igual que hemos constatado distintas finalidades como telespectador en un mismo sujeto, en este caso la diversión o entretenimiento no estarían reñidas con el aprendizaje. Por ello, compartimos la hipótesis de otros autores (Medrano et al, 2007), entre otros, de que "los propios contenidos televisivos son fuente de aprendizaje" (Medrano et al, 2007, p. 307). De ahí, la importancia de educar en y con los medios de comunicación.

Todo esto nos lleva a una última reflexión, en nuestro caso, educativa. Puesto que confiamos en las posibilidades de la Institución Educativa, capaz de dar respuesta a las nuevas necesidades que exige el vivir en un mundo caracterizado por las tecnologías de la información y de la comunicación. Y para ello, un primer paso es aceptar la necesidad de educar en este medio, pero también servirnos de él como un recurso más que nos ayude en ese aprendizaje crítico. Sin embargo, no debemos dejar toda la responsabilidad a la escuela, sino que juegan un papel fundamental otros contextos, como el familiar, socio-comunitario y cultu- 
ral. De ahí, la necesaria coordinación entre organismos oficiales y las distintas productoras, pero también con las Instituciones Educativas y las familias. Puede parecer utópico dicha cooperación, no obstante, la vida es compleja, y la televisión forma parte de dicha vida, y es por ello que una educación en dicho medio es fundamental para conseguir el gran reto de la educación: conseguir la emancipación y libertad, en todos los aspectos, de los individuos.

\section{Referencias bibliográficas}

Aguaded, J. I. (2000). Televisión y telespectadores. Huelva: Grupo Comunicar.

Aldea Muñoz, S. (2004). "La influencia de la 'nueva televisión' en las emociones y en la educación de los niños". Revista de Psiquiatría y Psicología del Niño y del adolescente, 4 (2), 145-159.

Bermejo, J. (2005). Narrativa audiovisual. Investigación y aplicaciones. Madrid: Pirámide.

Buckingham, D. (1987). Public Secrets. Eastenders and its Audience. Londres: BFI.

Ferrés, J. (1996). Televisión subliminal. Barcelona: Paidós.

Ferrés, J. (2005). “Características, géneros y efectos de los mensajes televisivos”. Textos de Didáctica de la Lengua y de la Literatura, 38, 18-25.

França Rocha, M. E. (2001). La contribución de las series juveniles de televisión a la formación de la identidad en la adolescencia. Análisis de contenido y de la recepción de la serie "Compañeros" (Antena 3). Tesis doctoral no publicada, Universidad Autónoma de Barcelona.

Freixas, A. (Dir.) (2000). "Creencias y aspiraciones profesionales y vitales en la adolescencia. Publicidad televisiva y aspectos de género". Instituto de Estudios de la Mujer de la Universidad de Granada.

González, J. (1999). El discurso televisivo: espectáculo de la posmodernidad. Madrid: Catedra.

Hoffner, C. (1996). "Children's wishful identification and parasocial interaction with favorite televisión characters”. Journal of Broadcasting and Electronic Media, 40, 389-402.

INJUVE (2000). Informe juventud en España 2000. Avance de resultados. Madrid: Instituto Nacional de la Juventud.

Klitgaard, K. (1996). “Global teen soap go local: Beverly Hills, 90210 in Denmark”. Joung: Nordic Journal of Youth Research, 4, 3-20.

Medrano, C.; Palacios, S. y Aierbe, A. (2007). "Los hábitos y preferencias televisivas en jóvenes y adolescentes: Un estudio realizado en el País Vasco". Revista Latina de Comunicación Social, 62, $1-15$.

Medrano, C.; Cortés, A. y Palacios, S. (2007). "La televisión y el desarrollo de valores". Revista de educación, 342, 307-328.

Montero, Y. (2006). Televisión, valores y adolescencia. Barcelona: Gedisa.

Naval, C.; Lara, S.; Portilla, I. y Sadaba, Ch. (2002). Impacto de las Tecnologías de la Comunicación en la Juventud Navarra. Pamplona: Gobierno de Navarra, Instituto Navarro de Deporte y Juventud.

Newton, B. J. y Buck, E. B. (1985). "Televisión as significant other: Its relationship to self descriptors in five countries". Journal of Cross-Cultural Psychology, 16, 289-312.

Pasquier, D. (1999). La culture des sentiments. L'expérience télévisuelle des adolescents. Paris: Éditions de la Maison des sciences de l'homme.

Pérez Tornero, J. M. (2005). "TV, escuela y familia. En busca del equilibrio". Bayard Revistas, $12,1-5$. 
Rodríguez, E.; Navarro, J. y Megías, I. (2001). Jóvenes y medios de comunicación. La comunicación mediática entre los jóvenes madrileños. Madrid: Coedición de la Fundación de Ayuda contra la Drogadicción y el Instituto de la Juventud.

Rubin, A. M. y Perse, E. M. (1988). "Audience activity and soap opera involvement: A uses and effects investigation". Human Communication Research, 14, 246-68.

Sánchez García, M. F. (2003). "Funciones y técnicas de intervención para la transición y la inserción laboral". En A. Sebastián (Coord), Orientación profesional. Un proceso a lo largo de la vida (349-390). Madrid: Dykinson.

Sánchez Noriega, J. L. (1997). Crítica de la seducción mediática: comunicación y cultura de masas en la opulencia informativa. Madrid: Tecnos.

Vera Vila, J. (2005). "Medios de comunicación y socialización juvenil". Revista de Estudios de Juventud, $\mathrm{n}^{\mathrm{o}} 68,19-31$.

Fecha de recepción: 04-02-2008

Fecha de revisión: 16-02-2009

Fecha de aceptación: 05-05-2009 